\title{
HIGH RESOLUTION PROTON ENERGY LOSS SPECTROSCOPY OF HIGH OVERTONE LEVELS OF POLYATOMIC MOLECULES
}

\author{
W. MARING and J. P. TOENNIES \\ Max-Planck-Institut für Strömungsforschung, W-3400 Göttingen, Germany
}

\begin{abstract}
In the inelastic scattering of nearly monoenergetic proton beams $(\cong 20 \mathrm{eV})$ from polyatomic molecules such as $\mathrm{CF}_{4}$ sharp peaks are observed corresponding to the excitation of very high overtone levels $(n \leq 14)$ of the infrared active modes. The collision mechanism leading to the high degree of excitation is described and an example from recent experiments is presented. A fit of the $\mathrm{CF}_{4}$ spectra provides new anharmonicity constants for the $v_{3}$-mode.
\end{abstract}

KEY WORDS: Proton energy loss, overtone levels, polyatomic molecules.

\section{INTRODUCTION}

Despite the long history of vibrational spectroscopy very little is known about the high lying vibrational levels of polyatomic molecules. On the other hand these levels are of great interest in connection with multiphoton excitation, ${ }^{1}$ mode selective chemistry, unimolecular reactions, and fundamental questions concerning the transition in spectral energy levels from regular to irregular and finally to a fully chaotic regime. ${ }^{2}$ Direct optical excitation is limited to overtones of about $n \leq 6$ because of a sharp decrease in transition probability with increasing $n .{ }^{3}$ Stimulated emission pumping has been used for a few systems but is not universally applicable., ${ }^{4,5}$ Photodissociation emission spectroscopy ${ }^{6}$ and fluorescence following collision uppumping in low temperature matrices ${ }^{7}$ have also been shown to populate high vibrational states for special molecules.

Here we review briefly some recent progress in implementing a new spectroscopy (PELS) which was introduced some years ago. ${ }^{8}$ In the initial experiments the energy loss distribution of monoenergetic protons was found to show a progression of sharp peaks up to very high levels of $n \leq 14$ in $\mathrm{CF}_{4} \cdot{ }^{8,9}$ Levene and Perry carried out a detailed analysis of the excitation process and were able to show that the protons preferentially excite the delocalized modes within the tensor split manifold of a given vibrational overtone level of a spherical top molecule. ${ }^{10}$ The goal of the new experiments has been to increase the resolution sufficiently to determine spectroscopic constants. 


\section{THE PELS METHOD}

The observations can be explained by a simple theory based on the forced harmonic oscillator model for which the classical and the exact quantum mechanical energy transfer are identical:

$$
\Delta E_{k}=\frac{1}{2}\left|\int_{\infty}^{\infty} \overrightarrow{\mathrm{F}}_{k}(t) \exp \left(-i \omega_{k} t\right) \mathrm{d} t\right|^{2}
$$

where $\overrightarrow{\mathrm{F}}_{k}(t)$ is the mass weighted force acting on the normal coordinate $Q_{k}$ of the $k^{t h}$ normal mode. For a non-polar molecule $\overrightarrow{\mathrm{F}}_{k}(t)$ is given in first approximation by

$$
\overrightarrow{\mathrm{F}}_{k}(t)=-\frac{\partial \mu}{\partial \mathrm{Q}_{k}} \vec{\epsilon}(\overrightarrow{\mathrm{R}}(t))
$$

where $\partial \mu / \partial \mathrm{Q}_{k}$ is the dipole moment derivative and $\vec{\epsilon}(\overrightarrow{\mathrm{R}}(t))$ is the electric field at the molecular calculated for a given trajectory $\mathrm{R}(t)$ (R is the distance between the proton and the center of the molecule). Thus for a straight line trajectory corresponding to small scattering angles

$$
\Delta E_{k}=\frac{1}{2 m}\left|\frac{\partial \mu}{\partial \mathrm{Q}_{k}}\right|^{2} \frac{e^{2}}{b^{4}}\left|\vec{\epsilon}\left(\omega_{k}\right)\right|^{2}
$$

where $e$ is the charge of the proton, $b$ the impact parameter and $\vec{\epsilon}\left(\omega_{k}\right)$ the Fourier component of the electric field. For $b=1.5 \AA \vec{\epsilon}(\vec{R}(t))$ can reach a maximum value of $6 \cdot 10^{8} \mathrm{~V} / \mathrm{cm}$ and the energy transfer for a small scattering angle collision of $\mathrm{D}^{+}$ions at $E=20 \mathrm{eV}$ is $\Delta E \simeq 2.0 \mathrm{eV}$ ! The forced oscillator model predicts, furthermore, that the transition probabilities to the various overtones are given by a Poisson distribution:

$$
P(0 \rightarrow n)=\frac{1}{n !} \epsilon^{-n} e^{-\epsilon}, \epsilon=\frac{\Delta E_{k}}{\hbar \omega_{k}}
$$

This simple model is surprisingly accurate in explaining the qualitative and even many of the quantitative features of the scattering experiments for molecules for which charge transfer is endothermic, e.g. $\mathrm{CF}_{4}, \mathrm{SF}_{6}$ and for $\mathrm{CH}_{4}$ at small scattering angles.

A few words of interpretation are appropriate. For one, $\Delta E_{k}$ is the mean energy transfer and should by no means be associated with the width of the experimental loss peaks. In a scattering experiment as in optical spectroscopy the final states are the stationary states of the system at long times $(t=\infty)$ after the proton has collided with the molecule. In the PELS experiment the line widths, in fact, are presently determined by the resolution of the apparatus only. Moreover the excitation in PELS is coherent since all the overtone levels are populated in a time of $10^{-15} \mathrm{sec}$ which is short compared to the vibrational period. Multiphoton excitation is an incoherent process since many successive optical excitations are involved. 


\section{APPARATUS AND EXPERIMENTAL RESULTS}

Figure 1 shows a schematic diagram of the PELS scattering experiment. The mass and energy selected proton beam with an energetic half width of $\Delta E=12-15 \mathrm{meV}$ at $E_{i}=20 \mathrm{eV}$ and a current of about $10^{6} \mathrm{ions} / \mathrm{sec}$ is crossed with a secondary nozzle beam of the target molecules. The protons scattered under small angles $0^{\circ} \leq \theta_{i} \leq 10^{\circ}$ are analyzed for their final energy distribution with a double hemispherical electrostatic analyzer similar to the one used in the incident beam for energy selection. Figure 2 shows an energy loss spectrum for the target molecule $\mathrm{CF}_{4}$. The energy distribution consists essentially of three parts:

1) a very intense elastic peak which is far off scale and due mostly to unscattered protons

2) a set of inelastic peaks with a mean energy transfer of about $25 \cdot 10^{-3} \mathrm{eV}$

3) a weak series of peaks with a maximum in the overall envelope at about $2.0 \mathrm{eV}$.

The inelastic peaks (2) are due to grazing collisions with relatively large impact parameters of about $4.5 \AA$. The energy transfer predicted by Eq. (3) agrees nicely with the maximum of this series of peaks. The second series of inelastic peaks is due to repulsive collisions which as a result of compensating long range forces also appear under small scattering angles. They have impact parameters of only about $1.5 \AA$ and according to Eq. (3) are expected to have a factor 81 greater energy transfer in good agreement with the observed value of $\Delta E=2.0 \mathrm{eV}$. The progression of peaks can be attributed to the $v_{3}$-mode since it has the largest value of $\partial \mu / \partial \mathrm{Q}$ of all the normal modes. Moreover it is gratifying to see that the distribution of peak intensities follows nicely the predicted Poisson distribution.

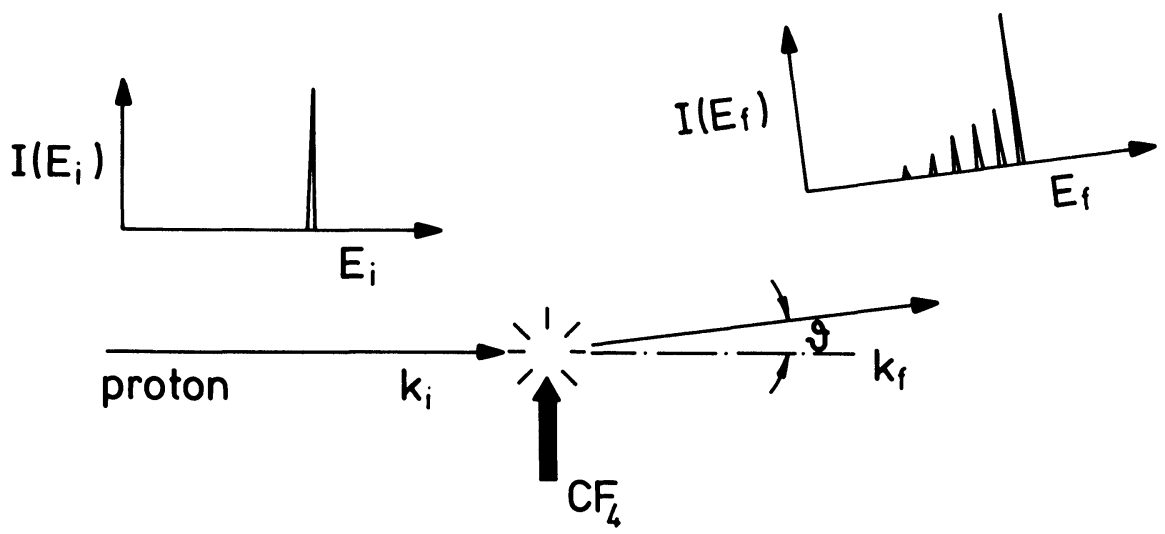

Figure 1 Schematic diagram of a proton energy loss scattering experiment. A nearly monoenergetic incident beam is scattered from a well collimated nozzle beam. The energy distribution of the scattered ions reveals the spectroscopic energy levels of the molecule. 


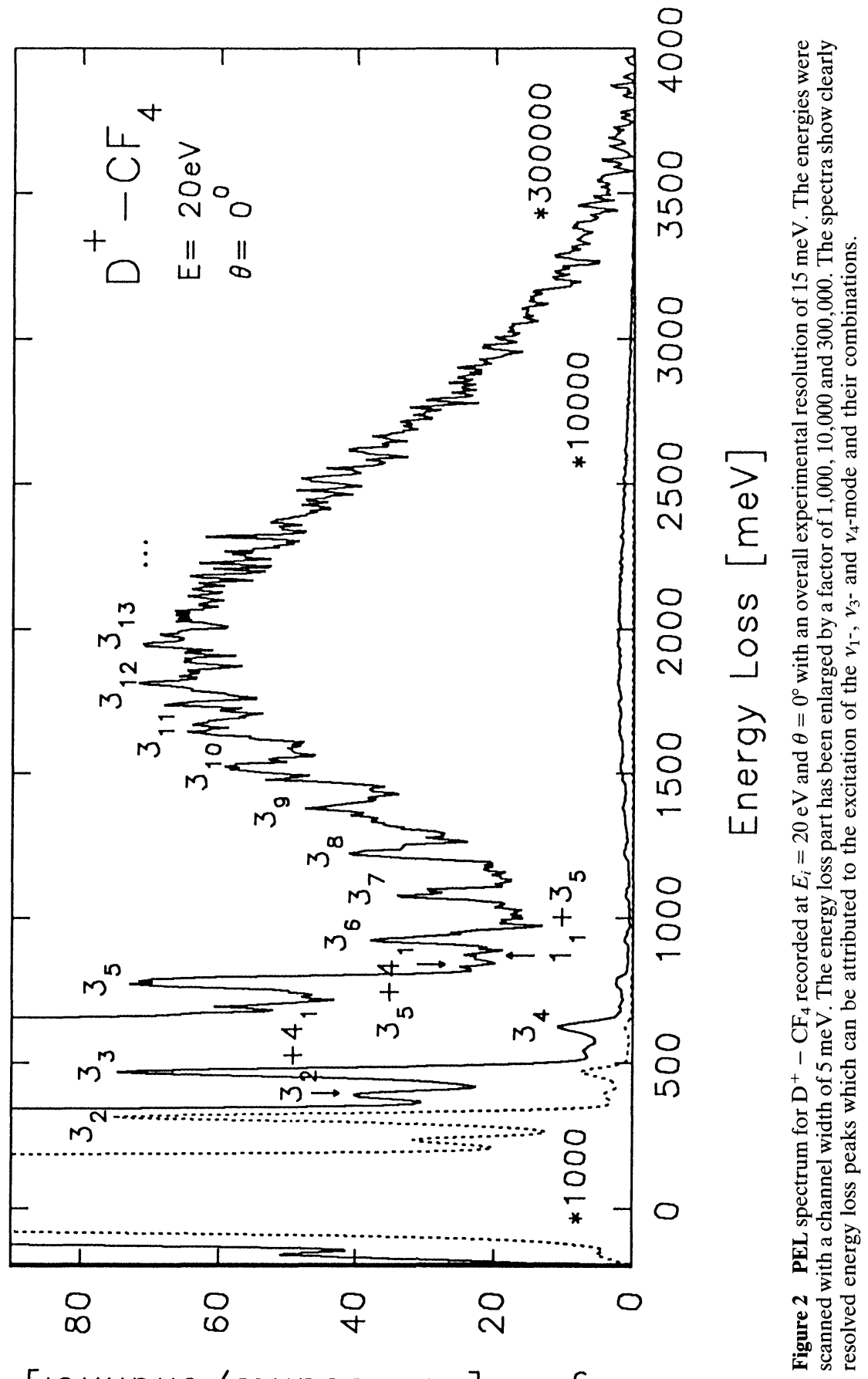

[iauuduo/sfunos ${ }_{9} \mathrm{OL} *$ ] IDUG!S 


\section{MOLECULAR SPECTROSCOPY}

In this brief introduction to the PELS method it is not possible to go into the full details of the spectroscopic interpretation, which has been discussed in detail in the paper by Levene and Perry. ${ }^{10}$ For spherical top molecules account must be taken of the fact that for the high overtone levels the large number of $n$ vibrational quanta in the triply degenerate $v_{3}$-mode can be distributed among the different possible modes in different ways. These can be classified as delocalized if the energy is uniformly distributed among the different oscillators or localized if all or most of the quanta are in one of the localized modes. Since the anharmonicity shift goes as $n^{2}$ the energy of the localized modes is shifted downwards more than for the delocalized modes. Thus each overtone level is spread over an increasingly wide band whose width increases roughly with $n$. Whereas optical spectroscopy couples preferentially to the local modes, PELS excites predominately the delocalized modes. This can be attributed to the rotation of the electric field direction by $180^{\circ}$ during the course of the collision.

In the well-known Hecht hamiltonian ${ }^{11}$ for spherical top molecules the overall shift in each overtone band is given by the following expression ${ }^{12}$

$$
\begin{aligned}
\mathrm{G}= & \sum_{i}\left[\omega_{i}\left(n_{i}+\frac{1}{2} d_{i}\right)+\sum_{k \geq i} \mathrm{X}_{i k l}\left(n_{i}+\frac{1}{2} d_{i}\right)\left(n_{k}+\frac{1}{2} d_{k}\right)\right. \\
& \left.+\sum_{k \geq i} \sum_{l \geq k} \mathrm{X}_{i k l}\left(n_{i}+\frac{1}{2} d_{i}\right)\left(n_{k}+\frac{1}{2} d_{k}\right)\left(n_{l}+\frac{1}{2} d_{l}\right)\right]+\ldots
\end{aligned}
$$

Note that the terms $\mathrm{X}_{i k}$ and $\mathrm{X}_{i k l}$ are weighted by $n^{2}$ and $n^{3}$, respectively, and therefore their influence increases with $n$ drastically even though the $\mathrm{X}$ themselves may be only small terms and have only a very weak influence on the spectra at lower values of $n$.

A spectrum for $\mathrm{CF}_{4}$ similar to the one shown in Fig. 2 has recently been fitted by taking full account on the Hecht hamiltonian including the overtone level splitting. ${ }^{13}$ The best fit yielded a new value of $X_{33}=-9.1 \pm 1 \mathrm{~cm}^{-1}$ which is much larger than the literature value of $-5.6 \mathrm{~cm}^{-1}$ obtained from a spectroscopic analysis of the much better resolved infrared spectra which extend only up to $n=2 .{ }^{10} \mathrm{The}_{\mathrm{CF}}$ spectrum in Fig. 2 was analyzed in a similar way and since the energy transfer is greater than in the earlier work (by using $\mathrm{D}^{+}$instead of $\mathrm{H}^{+}$) it was possible to determine a value of $\mathrm{X}_{333}=-0.05 \mathrm{~cm}^{-1}$, which is the first report of such a value. In this analysis the other tensor splitting and anharmonicity parameters were kept the same as determined in the infrared studies.

\section{References}

1. S. J. Smith and P. L. Knight (Eds.), Multiphoton Processes: Proceedings of the 4th International Conference on Multiphoton Processes, Boulder, Colorado (Cambridge University Press, 1988).

2. Th. Zimmermann, L. S. Cederbaum, H.-D. Meyer and H. Köppel, J. Chem. Phys. 91, 4446 (1987).

3. A. Amrein, H. R. Dübal, M. Lewerenz and M. Quack, Chem. Phys. Lett. 112, 387 (1984).

4. C. E. Hamilton, J. L. Kinsey and R. W. Field, Annu. Rev. Phys. Chem. 37, 493 (1986).

5. H.-L. Dai (Ed.), J. Opt. Soc. Am. B7, 1801 (1990).

6. D. Imre, J. L. Kinsey, A. Sinha and J. Krenos, J. Phys. Chem. 88, 3956 (1984). 
7. R. Disselkamp and G. E. Ewing, J. Phys. Chem. 93, 6334 (1989).

8. M. Noll and J. P. Toennies, Chem. Phys. Lett. 108, 297 (1984).

9. U. Gierz, M. Noll and J. P. Toennies, J. Chem. Phys. 83, 2259 (1985).

10. H. B. Levene and D. Perry, J. Chem. Phys. 84, 4385 (1986).

11. K. T. Hecht, J. Mol. Spectrosc. 5, 355 (1966).

12. The term $\mathrm{X}_{i k l}$ has not been included in previous work.

13. W. Maring, J. P. Toennies, R. G. Wang and H. B. Levene, subm. to Chem. Phys. Lett. 